satisfactory. Curiously enough the reduction in fertility produced by antiL.DH-X antibodies is owing mainly to embryonic death during cleavage and implantation. The fact that spermagglutinating but non-immobilising sera could prevent sperm penetration through cervical mucus in an in vitro test was reported by B. Boettcher (University of Newcastle, New South Wales). S. Shulman (New York Medical College) noted the occurrence in cervical mucus of sperm-immobilising antibodies active in the absence of complement. S. Isojima (Hyogo Medical College, Nishinomiya) described an improved microtechnique for detecting sperm-immobilising antibodies in cervical mucus. The risks involved in the use of placental hormones and sperm antigens were considered critically by K. S. K. Tung (University of New Mexico, Albuquerque).

The foetus as a homograft received its share of attention, the feeling clearly being that the reasons for the lack of foetal rejection are still poorly understood. But further support was provided for the idea that genetic dissimilarity favoured implantation.

\section{Charting the cell cycle}

from $M$. Vicente

The Third European Workshop on the Cell Cycle sponsored by the European Cell Biology Organisation was held in Edinburgh on September 23-25.

Simpr.f. systems still seem to be leading the race for the understanding of the events constituting the cell cycle. Although the majority of the papers dealt with eukaryotic systems, the most interesting results were reported from studies of prokaryotes or relatively simple eukaryotes such as yeast and the slime mould Physarum polycephalum. These organisms share the property of being either single cells or plasmodia in which the life history of a growing individual is not heavily disturbed or dependent on the presence of other individuals or their metabolic products. Moreover all of them are suitable for biochemical and genetical studies.

M. Masters (University of Edinburgh) has produced strains of Escherichia coli in which a duplicate copy of the chromosomal origin of replication is carried on a plasmid. Such strains have a significantly reduced number of chromosomes per cell mass and also divide at a proportionally larger size. These properties are consistent with the idea that there is a fixed amount of some substance required for the initiation of DNA replication at the chromo- some origin and that the presence of extra copies of this origin results in competition between the chromosome and the plasmid for initiation at each mass doubling.

Three communications by $\mathrm{P}$. Thuriaux et al., P. Fantes and P. Nurse (University of Edinburgh), gave a new view of the cell cycle in the fission yeast Schizosaccharomyces pombe obtained from the study of mutants in which progression through the cycle is blocked or altered at different stages. There is a close similarity between the cell cycle in Saccharomyces cerevisiae and that in $S$. pombe as described by Thuriaux $e t$ $a l$. Both cycles are in turn very similar to the $E$. coli cycle summarised at the meeting by W. D. Donachie (University of Edinburgh). The cycles in the three species have two independent pathways, one involving chromosome replication, both of which are required for the normal progression of the cell towards division. There is also evidence suggesting that the pathways comprise several sequentially dependent reactions.

Homeostatic control of cell size in $S$. pombe was observed by $P$. Fantes studying a thermosensitive mutant blocked at a stage before nuclear division at the restrictive temperature. The treatment does not seem to disturb cell growth in other ways and allows cell elongation to proceed. In these circumstances a negative correlation between the length of a cell at division and the duration of the following cycle is observed once the cells are shifted back to the permissive temperature. Some of the processes leading to division are more dependent on time than on size, however, as no matter how long the cells become before the temperature release they always require a minimum time before they can divide.

That initiation of DNA synthesis can be controlled by size while nuclear division is controlled by time was suggested by Nurse when interpreting his observations in a $S$. pombe mutant in which mass at division is half the wild type value and DNA synthesis is displaced to a later stage of the cycle while timing of nuclear division remains unchanged. The simultaneous functioning of timing and size controls is one more aspect in which the cell cycle in $S$. pombe is analogous to that of $E$. coli.

Replicating DNA fibres of $S$. cerevisiae have been made visible by autoradiography, after pulses of radioactive uracil, by $\mathrm{D}$. H. Williamson and T. D. Petes (National Institute for Medical Research, London). They find that DNA replication proceeds bidirection. ally in most of the replicons starting from several replication origins in each fibre, as in other eukaryotes. Therefore most of the replicons seem to initiate synthesis very early in the $S$ period as replication becomes resistant to inhibition of protein synthesis once the $S$ period has started.

A quite different replication pattern is present in Physarum polycephalum in which several protein-dependent initiation events are detected by cycloheximide inhibition. Studying replication of DNA during the step that takes place $30 \mathrm{~min}$ after mitosis, F. Haugli (University of Troms $\varnothing$ ) has isolated, after short pulses of ${ }^{3} \mathrm{H}$-thymidine, $7 \mathrm{~S}$ DNA fragments very similar to the $E$. coli Okazaki pieces. These fragments are gradually chased into longer intermediates by a process that closely resembles the joining by ligase in E. coli.

Both R. Braun and V. M. Vogt (University of Berne) and $\mathrm{H}$. W. Sauer et al. (University of Konstanz) suggested that the DNA that codes for ribosomal RNA in Physarum is contained in a structure that resembles morphologically a bacterial plasmid. In addition Braun and Vogt have found that replication of this rDNA occurs at random during G2, a mode of replication that is consistent with that found in plasmids.

Reports on Physarum presented at the workshop show that studies on this organism are in a state comparable with that of $E$. coli in the early 1960 s - that can be taken optimistically or pessimistically depending on how one views it.

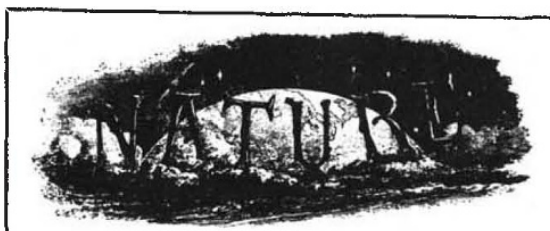

\section{A hundred years ago}

SIXTH REPORT OF THE SCIENCE COMMISSION

THREE times within the last twelve I years a Royal Commission has reported on the science teaching of our higher schools. In 1864 the Public Schools Commission announced that from the largest and most famous schools of all it was practically excluded. In 1868 the Endowed Schools Commission declared that the majority of school teachers had accepted it as part of their school work. The Science Commissioners of 1875, in their Sixth Report, on Science Teaching in Schools. testing this statement by inquiry, reports that of 128 endowed schools examined by them not one-half has even attempted to introduce it, while of these only 13 possess a laboratory, and only 10 give to the subject as much as four hours a week. And this statement is curiously illustrated by the statistics of the recent Oxford and Cambridge School Examination, which show that out of 461 candidates for certificates from 40 first-class schools. while 438 boys took up l.atin, 433 Greek, 455 Elementary Mathematics. 305 History; only 21 took up Mechanics, 28 Chemistry. 6 Botany. 15 Physical Geography.

From Nature, 12, 549, October 28, 1875. 